\title{
The Effect of Seoritae Extract in Men with Mild to Moderate Lower Urinary Tract Symptoms Suggestive of Benign Prostatic Hyperplasia
}

\author{
Woong Jin Bae, ${ }^{1,2}$ Hyo Jung Park, ${ }^{2}$ Hye Cheong Koo, ${ }^{3}$ Do Ram Kim, ${ }^{3}$ \\ U-Syn Ha, ${ }^{2}$ Kang Sup Kim, ${ }^{2}$ Su Jin Kim, ${ }^{2}$ Hyuk Jin Cho, ${ }^{2}$ Sung Hoo Hong, ${ }^{2}$ Ji Youl Lee, ${ }^{2}$ \\ Sung Yeoun Hwang, ${ }^{3}$ and Sae Woong Kim ${ }^{1,2}$ \\ ${ }^{1}$ Catholic Integrative Medicine Research Institute, College of Medicine, The Catholic University of Korea, \\ Seoul 137-701, Republic of Korea \\ ${ }^{2}$ Department of Urology, College of Medicine, The Catholic University of Korea, Seoul 137-701, Republic of Korea \\ ${ }^{3}$ Korea Bio Medical Science Institute, Seoul, Republic of Korea \\ Correspondence should be addressed to Sae Woong Kim; ksw1227@catholic.ac.kr
}

Received 16 December 2015; Revised 11 April 2016; Accepted 19 May 2016

Academic Editor: Raffaele Capasso

Copyright (C) 2016 Woong Jin Bae et al. This is an open access article distributed under the Creative Commons Attribution License, which permits unrestricted use, distribution, and reproduction in any medium, provided the original work is properly cited.

\begin{abstract}
We evaluated the effects of Seoritae extract (SE) on mild to moderate lower urinary tract symptoms (LUTS) suggestive of benign prostatic hyperplasia (BPH). Seventy-six subjects with mild to moderate LUTS suggestive of BPH were prospectively recruited from the urology outpatient clinic and assigned to either SE ( $4200 \mathrm{mg}$ or 6 tablets 3 times a day) or matching placebo. The primary outcome variable, the International Prostatic Symptom Score (IPSS), was evaluated at baseline and at 4 and 12 weeks. Postvoid residual volume (PVR), maximum urine flow rate $\left(Q_{\max }\right)$, and prostate-specific antigen (PSA) levels were evaluated. IPSSs decreased significantly from baseline to 12 weeks within the SE group. Significant improvements in IPSS voiding scores at 4 and 12 weeks were also observed in the SE group compared to the placebo group. IPSS storage and quality of life scores were also significantly decreased at 12 weeks in the SE group. There was no change in $Q_{\max }$ or PVR in both groups after 12 weeks. Administration of SE for 12 weeks led to significant improvements in LUTS, and it can be concerned as a reasonable and safe alternative for men with mild to moderate LUTS.
\end{abstract}

\section{Introduction}

Benign prostatic hyperplasia (BPH), a progressive enlargement of the prostate gland, is an age-related process and highly prevalent among elderly men. The molecular mechanisms associated with the etiology of $\mathrm{BPH}$ have not yet been fully elucidated [1]. However, the prevalence of $\mathrm{BPH}$ histologically, macroscopically, and clinically increases with age and it is well recognized that testis and aging are risk factors for $\mathrm{BPH}$ [2]. Oxidative stress, considered to be a cause of aging, is also thought to play a role in the development of $\mathrm{BPH}[3,4]$. Hence, it is hypothesized that antioxidant reactions to remove free radicals or inhibit their generation may prevent aging as well as progression of $\mathrm{BPH}$ [5].

$\mathrm{BPH}$ usually occurs concurrently with lower urinary tract symptoms (LUTS) in aging men and is typically treated with pharmacological therapies, minimally invasive procedures, or surgery. Phytotherapy has recently been used to treat LUTS worldwide because of the many side effects of pharmacological therapies and surgical procedures. Numerous studies have shown that nutritional supplements and herbal medicines can have anti-inflammatory, estrogenic, and antiandrogenic effects owing to decreasing levels of sexual hormones binding globulin; inhibiting growth factor-stimulated proliferation of prostatic cells, lipoxygenase, aromatase, alphaadrenoceptors, and 5-alpha-reductase; and neutralizing free radicals [6].

Seoritae is a type of black soybean (Glycine max (L.) Merr.) grown in Korea. Unlike other black soybeans, the inside of Seoritae has a bluish color. It is a traditional Korean food that also offers health-promoting effects due to its 
high isoflavone and anthocyanin levels. In our previous study, we proposed that the oxidative stress mechanism is related to $\mathrm{BPH}$ progression and that anthocyanin is a potent antioxidant that can decrease prostate volume and prevent $\mathrm{BPH}$ progression $[7,8]$. We also demonstrated its inhibitory effect on 5-alpha-reductase and showed that the antioxidant properties of isoflavones and anthocyanin in Seoritae extract (SE) could help to prevent BPH occurrence and progression in rats [9].

Thus, we performed a placebo-controlled study to evaluate the clinical effects and safety of SE in human subjects with LUTS suggestive of BPH [10]. We hypothesized that SE would be beneficial in reducing LUTS.

\section{Materials and Methods}

2.1. Study Design. This study was conducted at the Catholic University of Korea, Seoul St. Mary's Hospital, in South Korea between December 2013 and August 2014. The study protocol was approved by the institutional review board of the Seoul St. Mary's Hospital of the Catholic University of Korea (KC13HISI0652).

Sample size was estimated from a previous drug study that evaluated International Prostatic Symptom Score (IPSS) differences between two groups as its primary outcome [11]. A mean IPSS difference of 2.9 between the two groups was used to calculate the sample size in this study. A total of 42 subjects in each group were needed for $80 \%$ power at a 0.05 significance level, assuming a $15 \%$ dropout rate.

Men between 50 and 80 years of age with BPH/LUTS for $\geq 6$ months and IPSS $\geq 8$ and $\leq 19$ were recruited if they either had never received treatment (phytotherapeutic agents, alpha-adrenergic blocking agents, or 5-alphareductase inhibitors) or had stopped treatment $\geq 2$ weeks prior to enrollment. Exclusion criteria were severe LUTS, defined as IPSS $\geq 20$; known hypersensitivity to SE; urinary symptoms due to known causes other than $\mathrm{BPH}$, including urinary tract stones, urethral strictures, urinary tract infections, primary kidney disease, neurogenic bladder, and prostatitis; diagnosed prostate or bladder cancer; previous transurethral prostatectomy; severe cardiovascular disorder; or hepatic disorder. Subjects with uncontrolled psychiatric disorders or senile dementia were also excluded. Subjects with PSA $\geq 4.0 \mathrm{ng} / \mathrm{mL}$ were excluded, but those with PSA 4.0-10.0 ng/mL without malignant tumors proven by prostate biopsy were eligible for enrollment.

2.2. Study Supplement. The SE used in our study was produced using the following method: Seoritae $(200 \mathrm{~kg})$ samples were extracted with 1,600 L of $30 \%$ ethanol for 3 hours at 90 $100^{\circ} \mathrm{C}$. The solution was then filtered twice through a $50 \mu \mathrm{m}$ and $1 \mu \mathrm{m}$ filter and concentrated in a vacuum evaporator $\left(60^{\circ} \mathrm{C}\right)$ to 70 brix. The residual solvent was removed from the $\mathrm{SE}$ with a drying machine for 18 hours at $60^{\circ} \mathrm{C}$. The resulting powder was then stored in a plastic bag until use.

Few guidelines are available on the appropriate dose and duration of SE administration for subjects with LUTS [12]. Jang et al. [9] administered SE (SE 1 dose $228 \mathrm{mg} / \mathrm{kg}$ and SE 2 dose $457 \mathrm{mg} / \mathrm{kg}$ ) to rats, and both SE groups had
TABLE 1: HPLC analysis of isoflavones and anthocyanin content $(\mathrm{mg} / \mathrm{g})$ of Seoritae extract. Triplicate samples of 3 lots of the Seoritae extract were analyzed by HPLC.

\begin{tabular}{lccc}
\hline Content & \multicolumn{3}{c}{ Seoritae extract } \\
\hline Daidzin* & $0.64 \pm 0.02$ & $0.64 \pm 0.01$ & $0.65 \pm 0.02$ \\
Genistin* & $0.68 \pm 0.02$ & $0.67 \pm 0.01$ & $0.68 \pm 0.02$ \\
Glycitin* & $0.10 \pm 0.00$ & $0.10 \pm 0.00$ & $0.11 \pm 0.00$ \\
Daidzein & $0.02 \pm 0.00$ & $0.02 \pm 0.00$ & $0.02 \pm 0.00$ \\
Genistein & $0.01 \pm 0.00$ & $0.01 \pm 0.00$ & $0.01 \pm 0.00$ \\
Glycitein & $0.04 \pm 0.00$ & $0.04 \pm 0.00$ & $0.04 \pm 0.00$ \\
\hline Total & $1.50 \pm 0.04$ & $1.49 \pm 0.02$ & $1.51 \pm 0.05$ \\
\hline Anthocyanin (cyanidin- & $0.15 \pm 0.00$ & $0.15 \pm 0.00$ & $0.15 \pm 0.00$ \\
3-O-glucoside) & & & \\
\hline
\end{tabular}

${ }^{*}$ The content of daidzin, genistin, and glycitin was changed into that of aglycone types of isoflavones using conversion factor of 1/1.6.

Mean \pm SD.

significantly reduced prostate weight and oxidative stress as well as increased apoptosis compared to the BPH-induced control group. Based on previous studies, $2400 \mathrm{mg}$ of SE was administered daily in the current study. The content of isoflavones and anthocyanin in SE was analyzed by high performance liquid chromatography (HPLC) using a Waters 2695 Preparation Module HPLC system with a Waters 996 Photodiode Array Detector at $260 \mathrm{~nm}$ or a Waters HPLC system with a 2487 Dual Wavelength Detector set at $520 \mathrm{~nm}$, respectively. The proportions of the isoflavones and anthocyanins in SE are shown in Table 1.

SE tablets $(700 \mathrm{mg}$ ) were prepared, each containing $400 \mathrm{mg}$ SE. The placebo contained flour with the same dose, shape, and color. Both tablets were produced by HANPOONG Pharm \& Foods Co, Ltd., in Jeollabuk-do, South Korea. The release criteria for SE tables are as follows: brown oblong shaped coated tablets with no off-taste and off-odor, the deviation of weight being within $5 \%$, the disintegration time being within 50 minutes, the absence of coliform bacteria and total aflatoxin, the deviation of content of isoflavones being within $20 \%$, the content of lead being less than $3.0 \mathrm{mg} / \mathrm{kg}$, the content of cadmium, total arsenic, and total mercury being less than $1.0 \mathrm{mg} / \mathrm{kg}$, the content of dichlorodiphenyl-trichloroethane and benzene hexachloride being less than $0.1 \mathrm{ppm}$ or $0.2 \mathrm{ppm}$, respectively, and the content of aldrin, dieldrin, and endrin being less than $0.01 \mathrm{ppm}$. The tablets were labeled and coded as study supplements. Tablet identity was not revealed to subjects who received the study supplement tablets. The total daily SE dose in this study was $2,400 \mathrm{mg}$ or 6 tablets.

2.3. Intervention. Subjects were assigned to receive either placebo or SE (2 tablets 3 times a day) for 12 weeks. All subjects were followed up at 4 and 12 weeks from the start of placebo or SE administration. At the first (screening) visit, a detailed clinical history was taken, including the medical history of present and past diseases and concomitant drug treatments. Prior to receiving the intervention, IPSSs were determined for all subjects. Other objective variables 


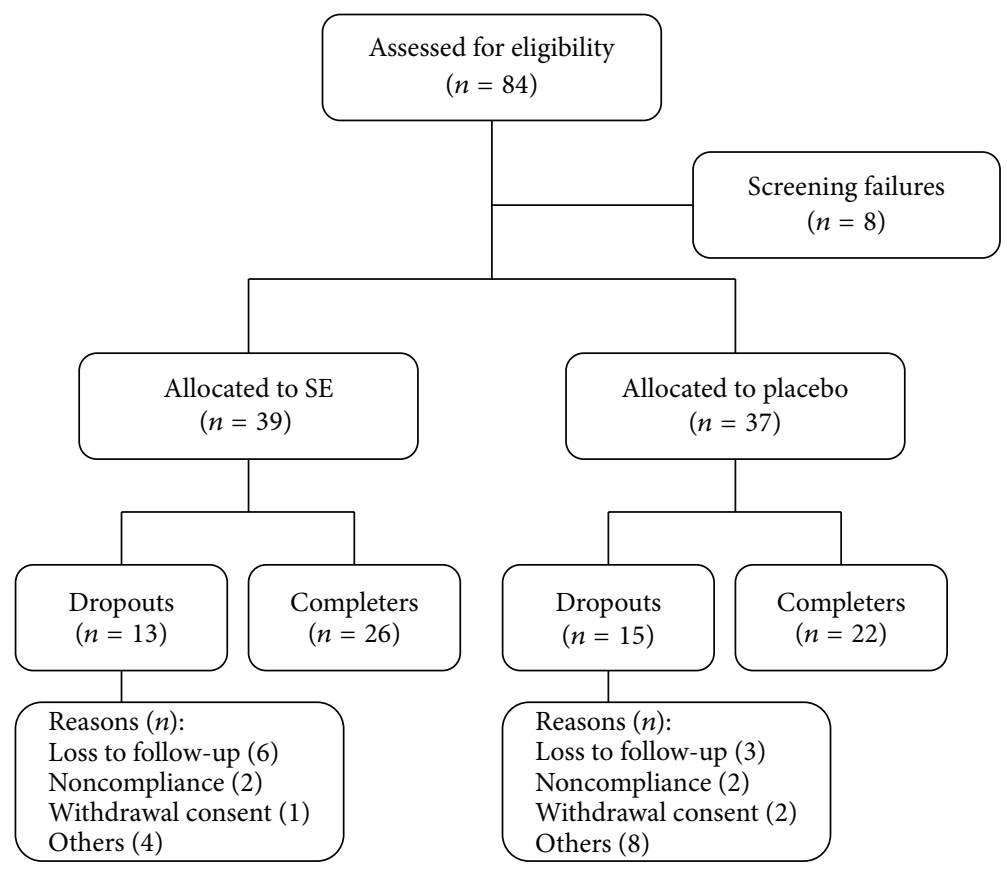

FIGURE 1: Subject disposition. SE, Seoritae extract.

included maximum urine flow rate $\left(Q_{\max }\right)$, postvoid residual volume (PVR), digital rectal examination, basic laboratory investigations (hematology, renal function tests, and urine analysis), and serum prostate-specific antigen (PSA) analysis. Compliance and drug-related side effects were assessed at visits 2 and 3 (at 4 and 12 weeks, resp., after starting treatment). Compliance was assessed by counting the number of tablets returned at next study visit.

2.4. Main Outcome Measures. The primary outcome measurement is the IPSS questionnaire form which is usually used to assess LUTS at the urology clinic. The IPSS consists of seven questions that assess frequency, nocturia, intermittency, urgency, incomplete emptying, weak stream, and straining with each graded with a score of $0-5$. At 12 weeks of treatment, the same efficacy variables were measured for comparison to previous measures. The secondary efficacy outcomes included objective $\left(Q_{\max }, P V R\right.$, and PSA) changes from baseline. The safety assessment included subject reports of adverse events and laboratory tests (hematology, clinical biochemistry, and urinalysis). Physical examinations and medical histories were assessed at study entry (baseline), 4 weeks, 12 weeks, and treatment discontinuation.

2.5. Statistical Analysis. The data are expressed as the mean (standard error of the mean), with $n$ indicating number of experiments. Mean differences between groups were compared by using independent $t$-test and Wilcoxon rank-sum test for normally and nonnormally distributed variables, respectively. $p$ values of 0.05 or less were considered statistically significant. All statistical analyses were performed using SAS version 9.3 (SAS Institute, Cary, NC, USA).
TABle 2: Demographic characteristics and outcome measures at baseline.

\begin{tabular}{lccc}
\hline & $\begin{array}{c}\text { SE group } \\
(n=39)\end{array}$ & $\begin{array}{c}\text { Placebo group } \\
(n=37)\end{array}$ & $\begin{array}{c}\text { Between groups } \\
p \text { value }\end{array}$ \\
\hline Age & $64.1 \pm 6.3$ & $65.1 \pm 7.9$ & 0.647 \\
Weight $(\mathrm{kg})$ & $69.1 \pm 7.7$ & $71.9 \pm 9.6$ & 0.158 \\
Height $(\mathrm{cm})$ & $168.7 \pm 5.8$ & $169.0 \pm 5.6$ & 0.848 \\
Comorbidity & $21(53.8)$ & $27(72.9)$ & 0.084 \\
Prostate volume $(\mathrm{cc})$ & $42.7 \pm 13.5$ & $34.0 \pm 14.2$ & 0.003 \\
IPSS & $12.9 \pm 3.6$ & $13.0 \pm 3.7$ & 0.925 \\
$\quad$ Voiding score & $8.1 \pm 3.0$ & $7.9 \pm 2.9$ & 0.875 \\
$\quad$ Storage score & $4.9 \pm 1.9$ & $5.1 \pm 2.1$ & 0.953 \\
IPSS QoL & $3.3 \pm 0.8$ & $3.4 \pm 0.8$ & 0.309 \\
Q & $13.3 \pm 5.9$ & $14.5 \pm 4.8$ & 0.410 \\
PVR & $32.2 \pm 29.3$ & $45.7 \pm 49.7$ & 0.484 \\
PSA & $1.7 \pm 1.4$ & $1.4 \pm 1.3$ & 0.065 \\
\hline
\end{tabular}

Mean \pm SD or $N(\%)$.

\section{Results}

A total of 84 patients were considered for enrollment. Eight subjects did not meet the inclusion criteria or declined to participate in the study. Seventy-six subjects who met the inclusion and exclusion criteria were assigned to two groups: 39 and 37 subjects in the SE and placebo groups, respectively. After 12 weeks of follow-up, 28 subjects were dropped for noncompliance $(n=4)$ or lost to follow-up $(n=9)$ (Figure 1$)$. Subject demographics and baseline characteristics are shown in Table 2. There were no significant differences between the two groups, except for prostate volume. 
TABLE 3: Mean changes in IPSS total and IPSS subscores from baseline to 12 weeks.

\begin{tabular}{lccc}
\hline & SE group & Placebo group & $\begin{array}{c}\text { Between groups } \\
p \text { value }\end{array}$ \\
\hline Baseline & & & \\
IPSS & $12.9 \pm 3.6$ & $13.0 \pm 3.7$ & 0.925 \\
$\quad$ Voiding score & $8.1 \pm 3.0$ & $7.9 \pm 2.9$ & 0.875 \\
$\quad$ Storage score & $4.9 \pm 1.9$ & $5.1 \pm 2.1$ & 0.953 \\
IPSS QoL & $3.3 \pm 0.8$ & $3.4 \pm 0.8$ & 0.309 \\
\hline 4 weeks & & & \\
IPSS & $11.7 \pm 4.7^{*}$ & $13.8 \pm 5.2$ & 0.080 \\
Voiding score & $7.2 \pm 3.2^{*}$ & $8.3 \pm 3.6$ & 0.231 \\
$\quad$ Storage score & $4.5 \pm 2.3$ & $5.5 \pm 2.7$ & 0.117 \\
IPSS QoL & $3.1 \pm 0.8$ & $3.5 \pm 0.8$ & 0.019 \\
\hline 12 weeks & & & \\
IPSS & $10.0 \pm 4.4^{*}$ & $14.9 \pm 5.7$ & 0.002 \\
Voiding score & $6.1 \pm 3.2^{*}$ & $8.9 \pm 4.1$ & 0.001 \\
$\quad$ Storage score & $3.8 \pm 2.0^{*}$ & $6.0 \pm 2.8$ & 0.001 \\
IPSS QoL & $2.5 \pm 1.1^{*}$ & $3.5 \pm 1.0^{*}$ & 0.001 \\
\hline Mean \pm SD, ${ }^{*} p<0.05$ changes from baseline. &
\end{tabular}

3.1. Primary Outcome. At the beginning of the trial, IPSSs in the SE and placebo groups were $12.9 \pm 3.6$ and $13.0 \pm$ 3.7, respectively ( $p=0.925$ ) (Table 3 ). IPSSs decreased significantly $(p<0.001)$ from baseline to 12 weeks $(10.0 \pm 4.4)$ within the SE group compared to the placebo group (13.0 \pm 3.7 at baseline and $14.9 \pm 5.7$ at 12 weeks) (Table 3$)$. At 12 weeks, the SE group achieved significant $(p<0.001)$ improvements in IPSS voiding $(6.1 \pm 3.2$ versus placebo $8.9 \pm 4.1)$ and storage scores $(3.8 \pm 2.0$ versus placebo $6.0 \pm 2.8)$. IPSS quality of life (QoL) scores decreased significantly from baseline to 12 weeks in the SE group $(p<0.001)$, but not in the placebo group. Differences in IPSS storage scores from baseline to 4 weeks in both groups were not significant. IPSS voiding and storage scores in the placebo group were also decreased from baseline to 12 weeks, but not significantly.

3.2. Secondary Outcome. The $Q_{\max }$ improved slightly in both groups, but the difference between groups was not significant. There was no change in PVR in either group at 12 weeks (Table 4). After 12 weeks, the mean PSA serum levels in the SE group were significantly increased compared with the placebo group. However, the differences of PSA serum levels compared with baseline were $0.2 \pm 0.5 \mathrm{ng} / \mathrm{mL}$ and $-0.1 \pm 0.5 \mathrm{ng} / \mathrm{mL}$ with the SE group and the placebo group, respectively $(p=0.099)$.

3.3. Safety and Tolerability. Among 76 recruited subjects, 5 (6.6\%) reported adverse events. There were no severe adverse events in either group during the study period. In the SE group, subjects complained of herpes zoster, constipation, and hematuria $(n=1,2$, and 1 , resp.), which were tolerable and did not require discontinuation of study supplement tablets. There were no clinically relevant differences in overall
TABLE 4: Results of urological examinations and serum PSA levels at baseline and 12 weeks.

\begin{tabular}{cccc}
\hline & $\begin{array}{c}\text { Seoritae extract } \\
(\text { mean } \pm \text { SD })\end{array}$ & $\begin{array}{c}\text { Placebo } \\
(\text { mean } \pm \text { SD })\end{array}$ & $\begin{array}{c}\text { Between groups } \\
p \text { value }\end{array}$ \\
\hline$Q_{\max }, \mathrm{mL} / \mathrm{s}$ & & & \\
Baseline & $13.3 \pm 5.9$ & $14.5 \pm 4.8$ & 0.410 \\
12 weeks & $14.3 \pm 6.2$ & $16.3 \pm 7.1$ & 0.232 \\
Difference & $1.01 \pm 4.7$ & $1.9 \pm 6.4$ & 0.693 \\
\hline PVR, mL & & & \\
Baseline & $32.2 \pm 29.3$ & $45.7 \pm 49.7$ & 0.484 \\
12 weeks & $43.9 \pm 57.6$ & $34.2 \pm 47.7$ & 0.457 \\
Difference & $11.8 \pm 52.9$ & $-11.5 \pm 48.4$ & 0.142 \\
\hline PSA, ng/mL & & & \\
Baseline & $1.7 \pm 1.4$ & $1.4 \pm 1.3$ & 0.065 \\
12 weeks & $1.9 \pm 1.8$ & $1.3 \pm 1.3$ & 0.012 \\
Difference & $0.2 \pm 0.5$ & $-0.1 \pm 0.5$ & 0.186 \\
\hline$Q_{\max }=$ maximum flow rate; PVR $=$ postvoid residual.
\end{tabular}

AE rates between groups $(p=0.359)$. In addition, there were no significant differences in vital signs and laboratory safety parameters in either group.

\section{Discussion}

The standard treatments for patients with symptomatic BPH include pharmacological therapies (alpha-1-blockers and 5alpha-reductase inhibitors) and surgery. Although these treatments are most effective for patients with moderate to severe $\mathrm{BPH}$, many patients have complained about their undesired side effects. The most common side effects of alpha-1-blockers include dizziness, postural hypotension, tachycardia, and retrograde ejaculation [13]. The side effects of 5-alpha-reductase inhibitors include erectile dysfunction, loss of libido, and ejaculation disorders [14]. Phytotherapeutic agents have become popular worldwide in patients with mild to moderate symptoms due to the side effects of the standard pharmacological therapies and surgical procedures. The premise of phytotherapy, that plant extracts might be as effective as pharmacologic agents without harmful side effects, makes it attractive to patients who prefer natural remedies [15]. Among phytotherapeutic agents, Serenoa repens, also known as saw palmetto, has become one of the 10 top-selling drugs in the United States; many urologists have recommended its use to treat voiding difficulties associated with $\mathrm{BPH}$ [16]. A randomized, double-blind, placebo-controlled study showed that administration of saw palmetto led to statistically significant improvements in urinary symptoms in men with LUTS compared with placebo [17]. However, a recent systematic review reported that Serenoa repens therapy, even at escalating doses, was not superior to placebo in improvement of symptoms, based on two high-quality clinical trials, one with a follow-up of 6 years [18].

Seoritae is a variety of black soybean with abundant isoflavones and anthocyanin. Anthocyanin is a water-soluble natural pigment that appears as red, purple, and blue in 
plants and belongs to the flavonoid parent class of molecules. It also acts as a powerful antioxidant with antiangiogenic, anticarcinogenic, and antioxidant effects [19-21]. Jang et al. showed that administration of anthocyanin to rats with induced prostatic hyperplasia resulted in reduced prostate weight and increased apoptosis [7]. Furthermore, anthocyanin significantly decreased apoptotic body count and antioxidant stress in a rat model of varicocele [8]. Isoflavone, a form of phytoestrogen, also possesses biochemical properties that can affect prostate physiology. It is abundant in soy, including primarily genistein and daidzein. Numerous studies have shown that isoflavones affect benign prostatic growth [22, 23] and 5-alpha-reductase activity [24-26]. Most studies have focused on the relationship between isoflavones and the prostate. In our previous study in a rat model of $\mathrm{BPH}$ [9], the SE group had significantly decreased prostate weight, oxidative stress, apoptosis, and 5-alpha-reductase. An in vitro study [27] showed that combined soy isoflavones were more efficacious than genistein or daidzein individually in inhibiting prostate epithelial cell growth.

Based on a similar mechanism, we suggested that SE would have an antioxidant effect and increase 5-alpha-reductase-activity due to the synergistic effects of anthocyanin and isoflavones in SE. These results may be the main mechanisms to decrease prostate weight and suppress prostate cell proliferation and these mechanisms may be effective in treating LUTS suggestive of BPH [9]. We found that subjects with $\mathrm{BPH}$ in the SE group had improved LUTS based on IPSS. Total IPSS as well as voiding and storage subscores significantly improved from baseline up to 12 weeks in the SE group. After 4 weeks of administration, IPSS was also significantly improved in the SE group compared to the placebo group.

SE did not significantly improve the mean $Q_{\max }$ or PVR compared with placebo. Our primary goal of treatment with SE is improvement in symptoms, not an increase in $Q_{\max }$. Therefore, the results of our study suggest that SE may be useful for relief of BPH symptoms although there was no change in $Q_{\max }$ or PVR after 12 weeks of administration in both groups. Serum PSA levels between the two groups were significantly different, but their change within groups from baseline to 12 weeks was not. A double-blinded, randomized trial with soy isoflavone supplementation conducted by Adams et al. [28] reported increased PSA levels in both groups (soy isoflavone versus no soy isoflavone), but the changes were not statistically significant. This result is also consistent with the previously mentioned study that reported that differences in serum PSA levels in the isoflavones and placebo groups were not significant at the 12th month [29]. There was a high adherence rate $(90.7 \%)$ with use of the study supplement tablets and no severe adverse events were reported.

This study had several limitations. First, our study was conducted with relatively few samples evaluated over a short period of time. This period was adequate to assess symptom relief but too short to observe any potential reduction of prostate size. For this reason, we did not measure prostate size at 12 weeks. In addition, we identified an unusual discrepancy in subjective outcomes between groups. For example, IPSS voiding and storage scores in the placebo group were also decreased from baseline to 12 weeks, but not significantly. Secondly, there was no restriction on soy food intake, which could be a confounding factor. The possibility for subconscious change in soy intake after entering the study also cannot be ignored. Further clinical studies with larger numbers of subjects and longer study periods are necessary to confirm these findings and determine longterm effects. Restriction or evaluation of dietary soy intake is also necessary in future studies. Thirdly, the baseline imbalance of the prostate volume between the SE and the placebo groups was shown $(42.8 \pm 13.5 \mathrm{~g}$ in SE group and $34.0 \pm 13.5 \mathrm{~g}$ in placebo group) (Table 2). More enlargement of prostate might be biased toward the null, though there was a weak correlation between IPSS and prostate volume [3032]. Therefore the difference of IPSS between both groups at 12 weeks might be underestimated. Finally, the SE used in this study was a standardized supplement according to wellestablished extraction guidelines, but its components could not be completely or chemically defined. Further study to assess its individual efficacy and identify other mechanisms is also necessary. In addition, it should be investigated with large subjects for a longer follow-up.

Administration of SE for 12 weeks led to statistically significant improvements in LUTS compared with administration of a placebo. This study suggests that SE can be concerned as a reasonable and safe alternative for men with mild to moderate LUTS, who choose not to take pharmacological therapies.

\section{Competing Interests}

The authors have no actual or potential conflict of interest associated with this work.

\section{Acknowledgments}

This work was supported by a grant from the NextGeneration BioGreen 21 Program (no. PJ011290012016), Rural Development Administration, Republic of Korea.

\section{References}

[1] W. Bushman, "Etiology, epidemiology, and natural history," Urologic Clinics of North America, vol. 36, no. 4, pp. 403-415, 2009.

[2] D. S. Coffey and P. C. Walsh, "Clinical and experimental studies of benign prostatic hyperplasia," Urologic Clinics of North America, vol. 17, no. 3, pp. 461-475, 1990.

[3] M. Ahmad, N. Suhail, T. Mansoor, N. Banu, and S. Ahmad, "Evaluation of oxidative stress and DNA damage in benign prostatic hyperplasia patients and comparison with controls," Indian Journal of Clinical Biochemistry, vol. 27, no. 4, pp. 385388, 2012.

[4] P. L. Minciullo, A. Inferrera, M. Navarra, G. Calapai, C. Magno, and S. Gangemi, "Oxidative stress in benign prostatic hyperplasia: a systematic review," Urologia Internationalis, vol. 94, no. 3, pp. 249-254, 2015.

[5] A. Kowald and T. B. L. Kirkwood, "Accumulation of defective mitochondria through delayed degradation of damaged 
organelles and its possible role in the ageing of post-mitotic and dividing cells," Journal of Theoretical Biology, vol. 202, no. 2, pp. 145-160, 2000.

[6] O. Allkanjari and A. Vitalone, "What do we know about phytotherapy of benign prostatic hyperplasia?" Life Sciences, vol. 126, pp. 42-56, 2015.

[7] H. Jang, U.-S. Ha, S.-J. Kim et al., "Anthocyanin extracted from black soybean reduces prostate weight and promotes apoptosis in the prostatic hyperplasia-induced rat model," Journal of Agricultural and Food Chemistry, vol. 58, no. 24, pp. 1268612691, 2010.

[8] H. Jang, S. J. Kim, S. M. Yuk et al., "Effects of anthocyanin extracted from black soybean seed coat on spermatogenesis in a rat varicocele-induced model," Reproduction, Fertility and Development, vol. 24, no. 5, pp. 649-655, 2012.

[9] H. Jang, W.-J. Bae, S.-M. Yuk et al., "Seoritae extract reduces prostate weight and suppresses prostate cell proliferation in a rat model of benign prostate hyperplasia," Evidence-Based Complementary and Alternative Medicine, vol. 2014, Article ID 475876, 7 pages, 2014.

[10] W. J. Bae, F. Bashraheel, S. W. Choi, S. J. Kim, B. I. Yoon, and S. W. Kim, "AB069. The effect of Seoritae extract in men with mildto-moderate lower urinary tract symptoms suggestive of benign prostatic hyperplasia," Translational Andrology and Urology, vol. 5, supplement 1, article AB069, 2016.

[11] L. S. Marks, M. C. Gittelman, L. A. Hill, W. Volinn, and G. Hoel, "Rapid efficacy of the highly selective $\alpha_{1 \mathrm{~A}}$-adrenoceptor antagonist silodosin in men with signs and symptoms of benign prostatic hyperplasia: pooled results of 2 phase 3 studies," Journal of Urology, vol. 189, no. 1, pp. S122-S128, 2013.

[12] A. Cassidy, "Potential risks and benefits of phytoestrogen-rich diets," International Journal for Vitamin and Nutrition Research, vol. 73, no. 2, pp. 120-126, 2003.

[13] S. Dutkiewics, "Efficacy and tolerability of drugs for treatment of benign prostatic hyperplasia," International Urology and Nephrology, vol. 32, no. 3, pp. 423-432, 2001.

[14] N. Mondaini, P. Gontero, G. Giubilei et al., "Finasteride $5 \mathrm{mg}$ and sexual side effects: how many of these are related to a nocebo phenomenon?" Journal of Sexual Medicine, vol. 4, no. 6, pp. 1708-1712, 2007.

[15] K. Dreikorn, "The role of phytotherapy in treating lower urinary tract symptoms and benign prostatic hyperplasia," World Journal of Urology, vol. 19, no. 6, pp. 426-435, 2002.

[16] M. Blumenthal, The ABC Clinical Guide to Herbs, Thieme Press, New York, NY, USA, 2003.

[17] G. S. Gerber, D. Kuznetsov, B. C. Johnson, and J. D. Burstein, "Randomized, double-blind, placebo-controlled trial of saw palmetto in men with lower urinary tract symptoms," Urology, vol. 58, no. 6, pp. 960-965, 2001.

[18] J. Tacklind, R. Macdonald, I. Rutks, J. U. Stanke, and T. J. Wilt, "Serenoa repens for benign prostatic hyperplasia," Cochrane Database of Systematic Reviews, vol. 12, Article ID CD001423, 2012.

[19] R. A. Jarred, S. J. McPherson, M. E. E. Jones, E. R. Simpson, and G. P. Risbridger, "Anti-androgenic action by red cloverderived dietary isoflavones reduces non-malignant prostate enlargement in aromatase knockout (ArKO) mice," Prostate, vol. 56, no. 1, pp. 54-64, 2003.

[20] M. Liu, X. Q. Li, C. Weber, C. Y. Lee, J. Brown, and R. H. Liu, "Antioxidant and antiproliferative activities of raspberries," Journal of Agricultural and Food Chemistry, vol. 50, no. 10, pp. 2926-2930, 2002.
[21] Z. Liu, J. Schwimer, D. Liu, F. L. Greenway, C. T. Anthony, and E. A. Woltering, "Black raspberry extract and fractions contain angiogenesis inhibitors," Journal of Agricultural and Food Chemistry, vol. 53, no. 10, pp. 3909-3915, 2005.

[22] S. J. Hong, S. I. Kim, S. M. Kwon, J. R. Lee, and B. C. Chung, "Comparative study of concentration of isoflavones and lignans in plasma and prostatic tissues of normal control and benign prostatic hyperplasia," Yonsei Medical Journal, vol. 43, no. 2, pp. 236-241, 2002.

[23] M.-Y. Chen, S.-C. Yan, C.-P. Yin et al., "Red clover isoflavones inhibit the proliferation and promote the apoptosis of benign prostatic hyperplasia stromal cells," Zhonghua Nan Ke Xue, vol. 16, no. 1, pp. 34-39, 2010.

[24] J. G. Lewis, S. Nakajin, S. Ohno, A. Warnock, C. M. Florkowski, and P. A. Elder, "Circulating levels of isoflavones and markers of $5 \alpha$-reductase activity are higher in Japanese compared with New Zealand males: what is the role of circulating steroids in prostate disease?" Steroids, vol. 70, no. 14, pp. 974-979, 2005.

[25] B. A. J. Evans, K. Griffiths, and M. S. Morton, "Inhibition of $5 \alpha$-reductase in genital skin fibroblasts and prostate tissue by dietary lignans and isoflavonoids," Journal of Endocrinology, vol. 147, no. 2, pp. 295-302, 1995.

[26] E. Pagano, M. Laudato, M. Griffo, and R. Capasso, "Phytotherapy of benign prostatic hyperplasia. A minireview," Phytotherapy Research, vol. 28, no. 7, pp. 949-955, 2014.

[27] T. E. Hedlund, A. Van Bokhoven, W. U. Johannes, S. K. Nordeen, and L. G. Ogden, "Prostatic fluid concentrations of isoflavonoids in soy consumers are sufficient to inhibit growth of benign and malignant prostatic epithelial cells in vitro," Prostate, vol. 66, no. 5, pp. 557-566, 2006.

[28] K. F. Adams, C. Chen, K. M. Newton, J. D. Potter, and J. W. Lampe, "Soy isoflavones do not modulate prostate-specific antigen concentrations in older men in a randomized controlled trial," Cancer Epidemiology Biomarkers and Prevention, vol. 13, no. 4, pp. 644-648, 2004.

[29] W. C. W. Wong, E. L. Y. Wong, H. Li et al., "Isoflavones in treating watchful waiting benign prostate hyperplasia: a doubleblinded, randomized controlled trial," Journal of Alternative and Complementary Medicine, vol. 18, no. 1, pp. 54-60, 2012.

[30] N. K. Bissada, A. E. Finkbeiner, and J. F. Redman, "Accuracy of preoperative estimation of resection weight in transurethral prostatectomy," Journal of Urology, vol. 116, no. 2, pp. 201-202, 1976.

[31] C. G. Roehrborn, C. J. Girman, T. Rhodes et al., "Correlation between prostate size estimated by digital rectal examination and measured by transrectal ultrasound," Urology, vol. 49, no. 4, pp. 548-557, 1997.

[32] E. I. Udeh, O. F. Ozoemena, and E. Ogwuche, “The relationship between prostate volume and international prostate symptom score in Africans with benign prostatic hyperplasia," Nigerian Journal of Medicine, vol. 21, no. 3, pp. 290-295, 2012. 


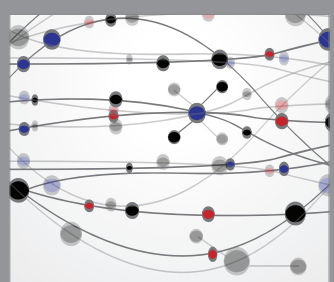

The Scientific World Journal
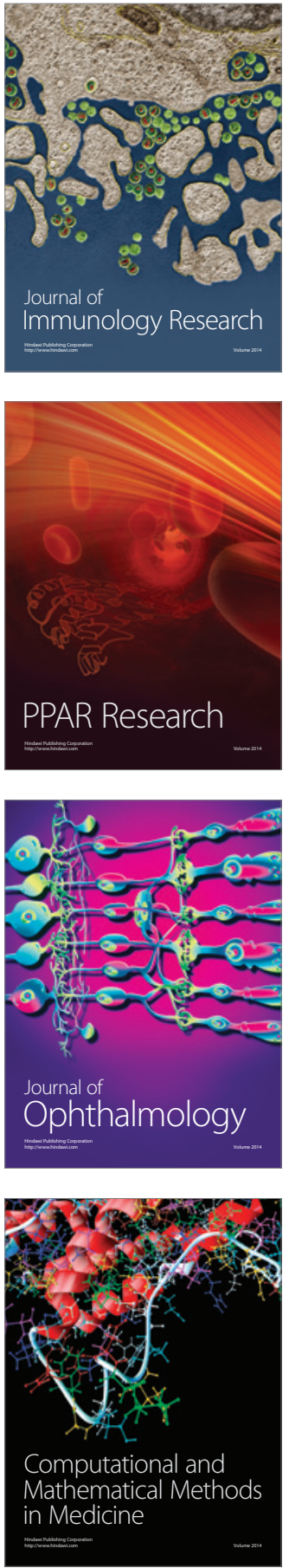

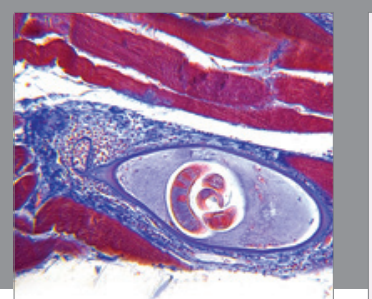

Gastroenterology Research and Practice

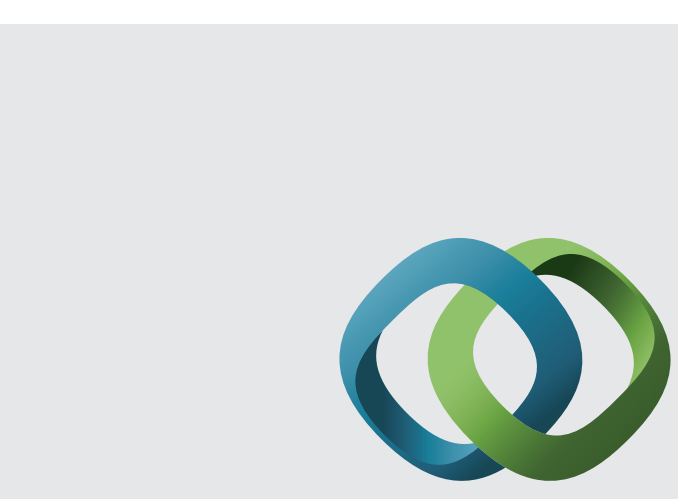

\section{Hindawi}

Submit your manuscripts at

http://www.hindawi.com
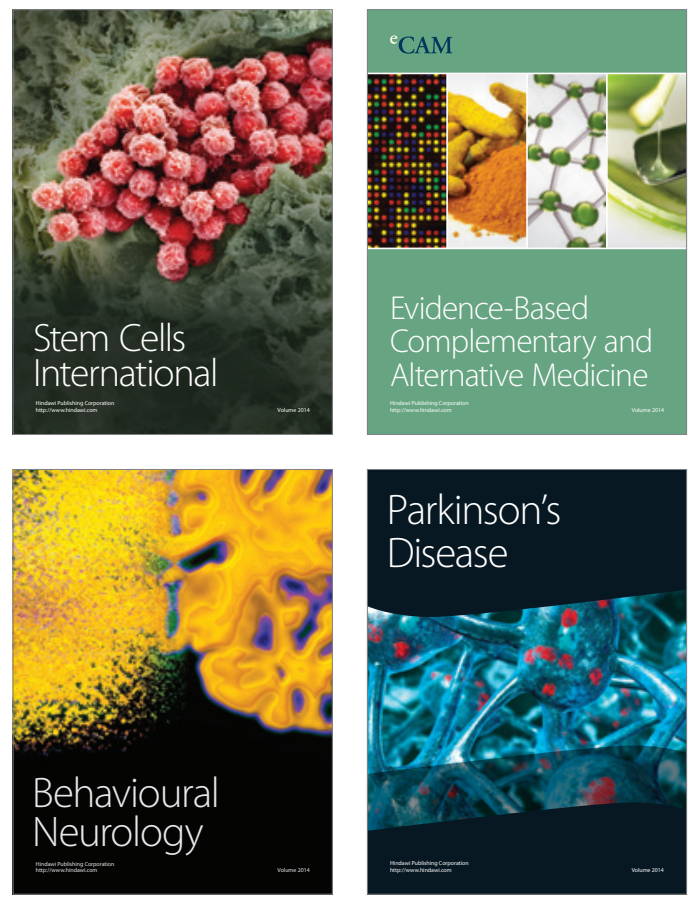
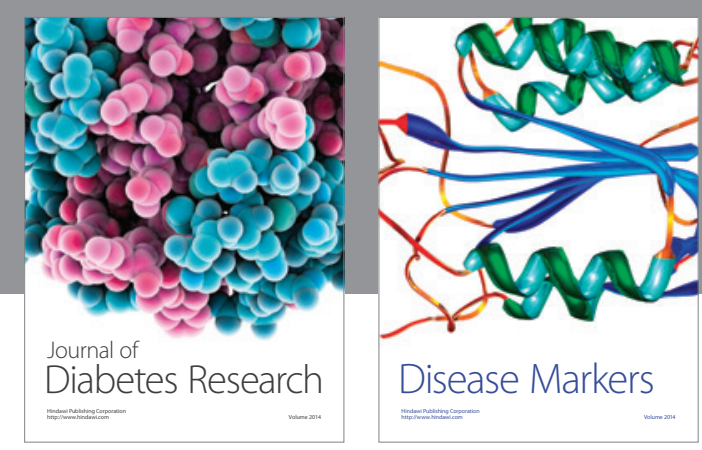

Disease Markers
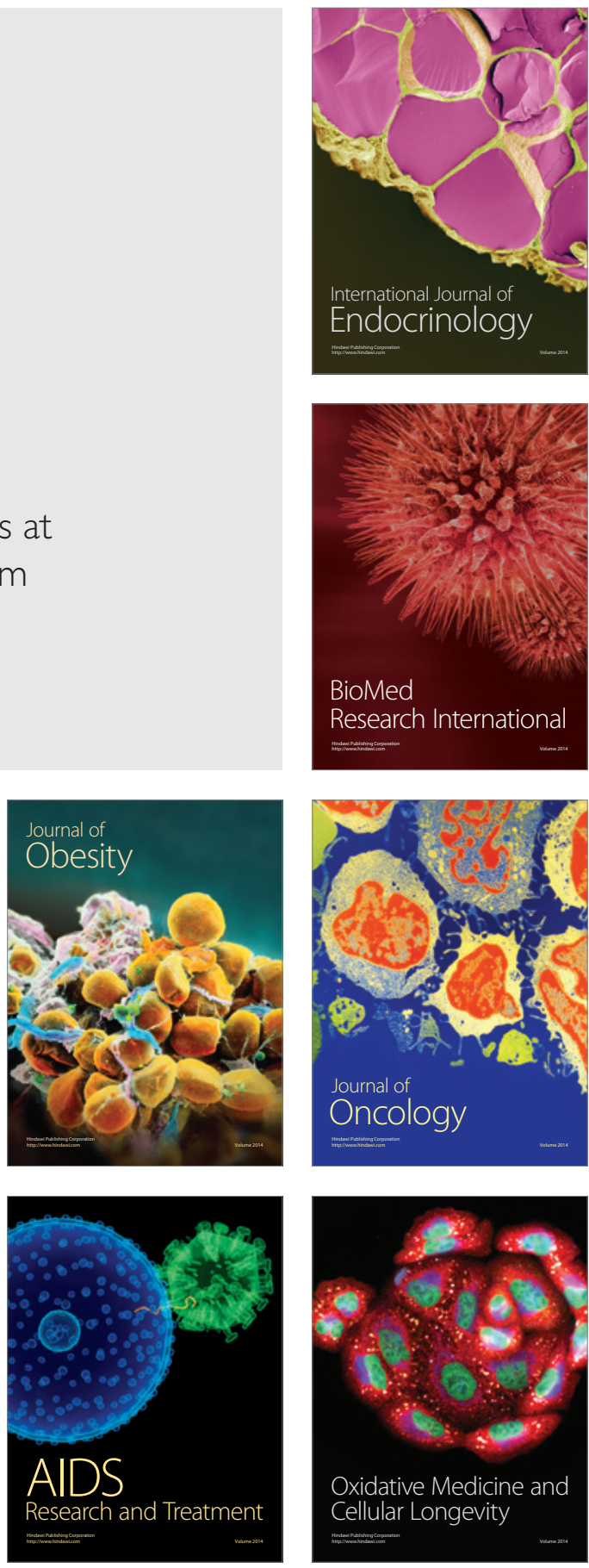